\title{
Treatment of uncomplicated gonorrhoea with single dose aztreonam
}

\author{
D T P EVANS,* A J R CROOKS,* C JONES,* R A HOLMAN, $\dagger$ AND S W PRICE $\dagger$
}

From the Departments of ${ }^{*}$ Genitourinary Medicine and $\dagger$ Medical Microbiology, Royal Infirmary, Cardiff

SUMMARY Infection with Neisseria gonorrhoeae was cleared in 61 men and 26 women at all sites (except in the pharynx of one male bisexual patient with urethral and pharyngeal gonorrhoea) after treatment with aztreonam as a single $1 \mathrm{~g}$ intramuscular injection. Aztreonam was well tolerated with no adverse effects. This monobactam antibiotic was effective against both penicillin sensitive and resistant strains.

\section{Introduction}

With the emergence of penicillinase producing Neisseria gonorrhoeae (PPNG) strains has come the need for $\beta$ lactamase stable compounds for treating gonorrhoea. Aztreonam, the first of a new class of $\beta$ lactam antibiotics, the monobactams, was developed specifically for treating infections due to aerobic Gram negative bacteria including the gonococcus. ${ }^{12}$ Aztreonam, given as a single $1 \mathrm{~g}$ intramuscular dose, has been shown to be effective in uncomplicated anogenital gonorrhoea, being effective against both penicillin sensitive and resistant strains in vitro. ${ }^{2}$ In vivo studies also show such efficacy. ${ }^{34} \mathrm{We}$ undertook this study to confirm the efficiency of aztreonam in treating gonorrhoea in Cardiff.

\section{Patients and methods}

The study was open in design and included male and female patients with acute uncomplicated genital or anorectal gonorrhoea, or both. Verbal informed consent was obtained from all patients, and the protocol was approved by the local ethical committee.

PATIENTS

We excluded patients from the study if they were aged under 18 or over 75 , had a history of an anaphylactic

Address for reprints: Dr D T P Evans, Department of Genitourinary Medicine, Royal Infirmary, Newport Road, Cardiff, South Wales

Accepted for publication 24 February 1986 reaction or other serious reaction to penicillins or cephalosporins, were pregnant or breast feeding, had a condition that required treatment with an anti-infective agent other than the study drug, had a neutrophil count of less than $1 \times 10^{9} / 1$, or had taken antibiotics, including metronidazole, in the previous 14 days or during treatment and follow up. (Concomitant local antifungal agents were permitted.) At entry to the study a routine clinical examination was carried out and clinical signs and symptoms recorded.

\section{MICROBIOLOGICAL METHODS}

Smears were taken from the urethra, cervix, rectum, or pharynx as appropriate and cultured on modified New York City medium to confirm gonococcal infection. Gram stained smears were examined in the clinic for the presence of Gram negative intracellular diplococci. A urine sample was obtained for urine analysis and culture. Production of $\beta$ lactamase was tested for by the acidometric method. Isolates were also tested for susceptibility to penicillin, cefuroxime, spectinomycin, and aztreonam using the disc diffusion method. $^{5}$

Patients were then treated with a single 1 dose of aztreonam injected into the gluteal muscle. They were asked to abstain from sexual intercourse and to attend follow up the next week (seven days) and one month (35 days) later. At follow up they were questioned about possible side effects and the tolerability of the injection. Cultures were taken from the urethra, cervix, and rectum of women at follow up.

Cultures were repeated at follow up visits to assess microbiological cure or failure. Patients who did not return for assessment within 21 days were excluded 
from analysis. Patients who had had sexual relations between the initial and follow up visits were excluded from analysis if the gonococcal infection was not eradicated, because of the possibility of reinfection.

\section{Results}

Of 102 patients enrolled in the study, 11 were excluded from analysis for the following reasons: six men because they had positive Gram films at entry, but subsequent culture was negative; four men and one woman because their cultures were still positive at follow up and they were known to have been reinfected.

This left a total of 91 evaluable patients for the study; 65 men and 26 women. Four of the men were homosexual and one was bisexual. The mean (SD) age of the patients was $24.5(6 \cdot 1)$ years. Four patients had concurrent infection with either Chlamydia trachomatis, Candida albicans, or Trichomonas vaginalis. The bisexual man, aged 25 , had a sore throat by the seventh day after treatment, which proved to be due to pharyngeal gonorrhoea.

Table I shows the sites from which $\boldsymbol{N}$ gonorrhoeae was isolated, and the subsequent cure rates. Of the 91

TABLE I Sites of infection with Neisseria gonorrhoeae and cure rates

\begin{tabular}{lcc}
\hline Site & No of patients & Cure rate (\%) \\
\hline Men: & & \\
Urethra & 64 & 100 \\
Rectum & 1 & 100 \\
Pharynx & 1 & 0 \\
Women: & & \\
Urethra & 26 & 100 \\
Cervix & 26 & 100 \\
Rectum & 1 & 100 \\
Pharynx & 0 & \\
\hline
\end{tabular}

NB some patients were infected at more than one site.

patients, 55 returned for follow up visits, but 36 failed to return for the second follow up. There was only one treatment failure, which occurred in a patient with both urethral and pharyngeal gonorrhoea. The infection was cleared from the urethra, but not from the pharynx.

The injection was well tolerated by 76 patients and moderately well tolerated by 14 , but one patient complained of pain. No adverse effects were reported. No abnormalities were noted on urine analysis, and no patients were taking concurrent medication for other indications.

In 81 patients the gonococcus was sensitive to all four agents tested. Ten patients had penicillin resistant
TABLE II Minimum inhibitory concentrations (MICs) of aztreonam for 91 strains of Neisseria gonorrhoeae by agar dilution

\begin{tabular}{lrllll}
\hline & \multicolumn{5}{c}{ No (\%) with MICs (mg/l) of: } \\
\cline { 3 - 6 } Clinical strain & $\begin{array}{c}\text { No of } \\
\text { cases }\end{array}$ & 0.125 & 0.3 & 0.4 & 0.5 \\
& 81 & $1(1)$ & $18(22)$ & $81(100)$ & $81(100)$ \\
Non-PPNG & 5 & 0 & 0 & $5(100)$ & $5(100)$ \\
PPNG & 5 & 0 & 0 & $5(100)$ & $5(100)$ \\
$\begin{array}{l}\text { Penicillin resistant } \\
\text { (non-PPNG) }\end{array}$ & 5 & & & & \\
\hline
\end{tabular}

strains, five of which were $\beta$ lactamase producers (PPNG). The minimum inhibitory concentration (MIC) of aztreonam for the PPNG strains was $0.4 \mathrm{mg} / 1$. For non-PPNG strains the MICs ranged from 0.125 $\mathrm{mg} / 1$ to $0.4 \mathrm{mg} / 1$ (table II). Of non-PPNG strains, $86.3 \%$ were sensitive to penicillin discs at a concentration of less than $0.06 \mathrm{mg} / \mathrm{l}$. All strains (PPNG and non-PPNG) were sensitive to aztreonam $30 \mu \mathrm{g}$ discs, spectinomycin $100 \mu \mathrm{g}$ discs, and cefurozime $30 \mu \mathrm{g}$ discs.

\section{Discussion}

Aztreonam is the first of a new class of monocyclic $\beta$ lactam antibiotics, the monobactams. It was developed specifically for the treatment of Gram negative bacterial infections and is highly stable to chromosomal $\mathbf{R}$ plasmid mediated $\boldsymbol{\beta}$ lactamase produced by aerobic Gram negative organisms. ${ }^{1}$ In contrast to recent cephalosporins, aztreonam does not induce $\beta$ lactamase production, ${ }^{6}$ which should therefore not be a major problem with this compound. Its range of activity in vitro toward Gram negative bacteria is comparable with that of the aminoglycosides and the third generation cephalosporins, and this has been confirmed in experimental Gram negative infections in animals. $^{7}$

Aztreonam also differs from the penicillins and cephalosporins in its low allergenicity, which is important in the treatment of patients who have a history of hypersensitivity to penicillin. ${ }^{89}$ Aztreonam conjugated to human serum albumin does not cross react in vitro with human IgG and IgE antipenicilloyl antibodies. ${ }^{810}$

Pharmacokinetic studies in man have shown that aztreonam has an elimination half life of $1 \cdot 3-2$ hours. It is widely distributed in body fluids and tissues and is eliminated primarily in the urine in an unchanged form. It is also secreted into the bile and is metabolised to a minor extent. ${ }^{11}$ After oral administration less than $1 \%$ is bioavailable, which means that aztreonam must be given intramuscularly. ${ }^{11}$ 
Aztreonam has been shown to penetrate the cerebrospinal fluid, ${ }^{11}$ and is effective against syphilis in experimental animals. ${ }^{12}$

The safety profile of aztreonam is similar to that of other $\beta$ lactam antibiotics, and the drug is well tolerated. ${ }^{13}$ With single dose treatment, six of 346 patients $(1 \cdot 7 \%)$ experienced adverse clinical effects. The most common reaction was nausea or vomiting, or both. No serious reactions have been reported. ${ }^{13}$

In our study all patients were cleared of their gonococcal infection at all sites, except for the pharynx of one male bisexual patient. Aztreonam was well tolerated and had no adverse effects. This therefore confirms the efficiency and tolerability of aztreonam as a single intramuscular injection for treating uncomplicated gonorrhoea. Penicillinase producing gonococci are now common in some centres, and it may be necessary to routinely use a $\beta$ lactamase stable antibiotic with the safety profile of a $\beta$ lactam agent, but with little or no cross sensitivity in patients allergic to penicillin or cephalosporin. Aztreonam promises to be such an antibiotic.

We thank E R Squibb and Sons, who supplied the aztreonam, and in particular Paul Woods (clinical trials manager at Squibb) for a critical review of the manuscript.

\section{References}

1. Sykes RB, Bonner DP, Bush K, Georgopapadakou NH, Wells JS. Monobactams-monocyclic beta-lactam antibiotics produced by bacteria. J Antimicrob Chemother 1981;9 (suppl E):1-16.

2. Percival A, Thomas E, Hart CA, Karayiannis P. In-vitro activity of monobactam, SQ 26,776 against Gram-negative bacteria. J Antimicrob Chemother 1981;8 (suppl E):49-55.

3. McClean KA, Harris JRW. Treatment of uncomplicated anogenital gonorrhoea with single dose aztreonam. Royal Society of Medicine International Congress and Symposium Series, 1985 No 89:69-74.

4. Gottleib A, Mills J. Effectiveness of aztreonam for the treatment of gonorrhoea. Antimicrob Agents Chemother 1985;27:270-1.

5. Stokes EJ, Ridgway GL. Clinical bacteriology, 5th ed. London: Edward Arnold, 1980:219-20.

6. Bush RB, Bonner DP. Monobactam antibiotics: history and development. Royal Society of Medicine International Congress and Symposium Series, 1985 No 89:4-24.

7. Bonner DP, Whitney RR, Baughn CO, Miller BH, Olsen SJ, Sykes RB. In-vivo properties of SQ 26,774. $J$ Antimicrob Chemother 1981;8 (suppl E):123-30.

8. Saxon A, Hassner A, Swabb EA, Wheeler B, Adkinson NF Jr Lack of cross-reactivity between aztreonam, a monobactam antibiotic and penicillin in penicillin-allergic subjects. $J$ Infect Dis 1984;149:16-22.

9. Adkinson NF, Saxon A, Spence MR, Swabb EA. Crossallergenicity and immunogenicity of aztreonam. Rev Infect Dis 1985;7 (suppl 4):613-621.

10. Adkinson NF, Swabb EA, Sugerman AA. Immunology of the monobactam aztreonam. Antimicrob Agents Chemother 1984;25:93-7.

11. Swabb EA. Review of the clinical pharmacology of the monobactam antibiotic aztreonam. Am $J$ Med 1985;78 (suppl 2A):11-8.

12. Lukehart SA, Baker-Zander SA, Holmes KK. Efficacy of aztreonam in treatment of experimental syphilis in rabbits. Antimicrob Agents Chemother 1984;25:390-1.

13. Newman TJ, Dreslinski GR, Tadros SS. Safety profile of aztreonam in clinical trials. Rev Infect Dis 1985;7 (supp 4):648-55. 